OPEN ACCESS

Edited by:

Dong-Yup Lee,

Sungkyunkwan University,

South Korea

Reviewed by:

Jianhua Wang,

Chinese Academy of Agricultural

Sciences, China

Fei Tao,

Shanghai Jiao Tong University, China

*Correspondence:

Sijing Jiang

jiangsijing@hubu.edu.cn

Guimin Zhang

zhangguimin@hubu.edu.cn;

zhangguimin6@hotmail.com

${ }^{\dagger}$ These authors have contributed

equally to this work

Specialty section:

This article was submitted to

Microbial Physiology and Metabolism,

a section of the journal

Frontiers in Microbiology

Received: 31 December 2020

Accepted: 17 February 2021

Published: 11 March 2021

Citation:

Li C, Jiang S, Du C, Lu Z, He N,

Zhou Y, Jiang S and Zhang G (2021)

High-Level Extracellular Expression

of a New $\beta$-N-Acetylg/ucosaminidase

in Escherichia coli for Producing

GlcNAc. Front. Microbiol. 12:648373.

doi: 10.3389/fmicb.2021.648373

\section{High-Level Extracellular Expression of a New $\beta$-N-Acetylglucosaminidase in Escherichia coli for Producing GIcNAc}

\author{
Congna Lit, Shun Jiang', Chao Du, Zhenghui Lu, Nisha He, Yuling Zhou, Sijing Jiang* \\ and Guimin Zhang*
}

State Key Laboratory of Biocatalysis and Enzyme Engineering, College of Life Sciences, Hubei University, Wuhan, China

$\mathrm{N}$-acetyl- $\beta$-D glucosamine (GlcNAc) is wildly used in cosmetics, nutraceuticals and pharmaceuticals. The traditional chemical process for GlcNAc production from chitin causes serious acidic pollution. Therefore, the enzymatic hydrolysis becomes a great promising and alternative strategy to produce GlcNAc. $\beta-N$-acetylglucosaminidase (NAGase) can hydrolyze chitin to produce GlcNAc. Here, a GH3 family NAGase encoding gene BINagZ from Bacillus licheniformis was expressed extracellularly in Escherichia coli guided by signal peptide PelB. The recombinant BINagZ presented the best activity at $60^{\circ} \mathrm{C}$ and $\mathrm{pH} 5.5$ with a high specific activity of $13.05 \mathrm{U} / \mathrm{mg}$. The BINagZ activity in the fermentation supernatant can reach $13.62 \mathrm{U} / \mathrm{mL}$ after optimizing the culture conditions, which is 4.25 times higher than optimization before. Finally, combining BINagZ with chitinase ChiA we identified before, chitin conversion efficiency to GlcNAc can reach $89.2 \%$ within $3.5 \mathrm{~h}$. In all, this study provided not only a high active NAGase, and a secreted expression strategy to reduce the cost of production, which is conducive to the industrial application.

Keywords: $\beta-\mathrm{N}$-acetylglucosaminidase, secretion, fermentation conditions optimization, enzymatic hydrolysis, GIcNAc

\section{INTRODUCTION}

GlcNAc is the basic component of biological polysaccharides, such as murein, hyaluronic acid, glycoproteins, proteoglycans, glycosaminoglycans (GAGs), and other connective tissue building blocks of mammals. It can also inhibit bacteria and fungi's growth, enhance human immunity, protect cartilage, bones and joints (Talent and Gracy, 1996). GlcNAc has been widely accepted as a health care product for delaying joint aging, cosmetic additives to improve wrinkles and pigmentation on the skin (Chen et al., 2010). As a drug, GlcNAc has also shown remarkable efficacy in the treatment of tumor, joint and intestinal inflammation (Shaoqing et al., 2014; Inokuma et al., 2016). Therefore, GlcNAc is in great demand in the market.

At present, GlcNAc is mainly prepared by chemical method, namely acid hydrolysis of chitin (Patil et al., 2013; García-Fraga et al., 2015), which cause serious environmental pollution. The enzymatic hydrolysis of chitin with mild reaction conditions, higher product purity and environmental friendliness becomes a great promising strategy for producing GlcNAc. The complete degradation of chitin to GlcNAc requires the synergistic action of chitinases 
and $\beta$-N-Acetylglucosaminidases (NAGases), that is, the colloidal chitin was hydrolyzed into chitin-oligosaccharides by chitinases, and further hydrolyzed into GlcNAc by NAGases (Du et al., 2019). Chitinase, such as ChiA, had achieved high-efficiency expression in our lab (Song et al., 2020). Therefore, it is essential to develop new NAGases with low cost for industrial use.

NAGases can be classified into four families of glycoside hydrolase $(\mathrm{GH}) 3,20,73$ and 84 , based on the character of amino acid sequence ${ }^{1}$. GH3 NAGases are usually distributed in several bacterial and metazoan cells, while members from the GH20 family are abundant in fungi and insects (Oliveira et al., 2018). Most GH20 NAGases are slightly alkaline (Zhou et al., 2017), which do not match the acid dissolution of colloidal chitin. NAGases of GH3 is mostly neutral and acidic, for example, the optimum $\mathrm{pH}$ of rNag3HWLB1 from Sphingobacterium sp. HWLB1 (Zhou et al., 2016) is 7.0, NahA from Symbiobacterium thermophilum (Ogawa et al., 2006) is 5.5, NagA from Streptomyces thermoviolaceus (Tsujibo et al., 1998) is 5.0 and NagA from Trichinella spiralis (Bruce and Gounaris, 2006) is 4.4, but their activity is generally low. It is well known enzymes' cost is a major impediment to the industrial application (Tsujibo et al., 1998). Therefore, the discovery of weak acid NAGases with high activity is the prerequisite for enzymatic preparation of GlcNAc, and the secretion expression of high level would lower the enzyme cost.

Several NAGases were already expressed inside the cells of E. coli, which required cell lysis during the purification (Zhou et al., 2016; Du et al., 2019). For ease of the process, BsNagZ secretory expression in Pichia pastoris with multiple copy strategy has been conducted, which takes 14 days per fermentation (Jiang et al., 2019). Since the activity of BsNagZ is low, we need to find new NAGases with higher activity and better thermal stability. After searching for NCBI database based on the amino acid sequence of BsNagZ, we selected several homologous NAGases to study further. NAGase from Bacillus licheniformis (BlNagZ) was expressed. Although $P$. pastoris has the advantage of secretory expression, the fermentation period is too long (Jiang et al., 2019). Hence, we try to express BlNagZ in E. coli to shorten the fermentation period. The popular pET vectors, which facilitate the fusion of target proteins to the signal peptide PelB, are usually the first choice for enzyme secretory expression in E. coli (Samant et al., 2014). PelB with the property of periplasmic targeting has successfully guided the secretion of several proteins from E. coli. For example, Chitosanase (CSN) from Aspergillus fumigatus was successfully expressed in E. coli followed by extracellular secretion under the guidance of PelB (Huang et al., 2016). Cutinase from Thermobififida fusca was expressed in E. coli and excreted through the mediation of the PelB (Chen et al., 2008, 2011).

In this study, a NAGase gene BlNagZ from B. licheniformis was expressed in E. coli and secreted out of the cells via PelB signal peptide. The recombinant BlNagZ showed the maximum activity of $13.05 \mathrm{U} / \mathrm{mg}$ at $\mathrm{pH} 5.5$ and $60^{\circ} \mathrm{C}$. The expression level was improved by 4.25 times after optimizing the fermentation conditions. Combined with the reported chitinase

${ }^{1}$ http://www.cazy.org/
(Song et al., 2020), recombinant BlNagZ can hydrolyze colloidal chitin to obtain GlcNAc with $89.2 \%$ conversion ratio within $3.5 \mathrm{~h}$.

\section{MATERIALS AND METHODS}

\section{Plasmid, Strains, Chemicals and Medium}

B. licheniformis WX-02 was offered by professor Chen Shouwen (Wei et al., 2010). The E. coli XL10-gold and BL21 (DE3) were purchased from Invitrogen (United States), and used for gene cloning and expression, respectively. The plasmid pET26b purchased from Stratagene was used as the expressive vector. The plasmids and strains were selected and maintained in LB medium containing $50 \mu \mathrm{g} / \mathrm{mL}$ kanamycin.

The DNA primers were synthesized by Sangon Biotech Co. (Shanghai, China). Restriction enzymes, Ex Taq DNA polymerase, $\mathrm{T}_{4}$ DNA ligase, and other related enzymes were obtained from TaKaRa (Dalian, China). The substrate 4Nitrophenyl-N-acetyl- $\beta$-D-glucosamine (pNP- $\beta$-D-GlcNAc) was purchased from Sigma-Aldrich (St. Louis, MO, United States). All the chemicals and reagents were analytical grade or chromatographic pure grade.

\section{Construction of the Expression Plasmid pET26b-BINagZ}

A pair of primers, BINagZ-F (5' TTCGGATCCGGTGAAAAA CATTAGAAAAACCGTTATTTTTG 3') and BlNagZ-R (5' AGT GCGGCCGCCTGATAACTTGGAACTTCCAATGTGATATT 3') were synthesized to amplify BlNagZ using the genomic DNA of $B$. licheniformis WX-02 as template. Then the PCR product was digested by restriction enzymes of BamHI and XhoI and cloned into the vector pET26b (Supplementary Figure 1). The recombinant plasmid, named pET26b-BlNagZ, was verified by DNA sequencing (Sangon Biotech Co., Shanghai, China).

\section{Expression and Purification of Recombinant BINagZ}

The recombinant plasmid pET26b-BlNagZ was transformed into E. coli BL21 (DE3). The recombinant strain BL21 (DE3)pET26b-BlNagZ was grown at $37^{\circ} \mathrm{C}$ overnight. Then the culture was transferred into $50 \mathrm{~mL}$ TB medium containing $50 \mu \mathrm{g} / \mathrm{mL}$ kanamycin, and grown at $37^{\circ} \mathrm{C}$. When the $O D_{600}$ of the culture reached 3.0, IPTG was added to a final concentration of $0.5 \mathrm{mM}$ to induce protein expression. After $32 \mathrm{~h}$, the fermentation supernatant was collected, and the target protein was detected by SDS-PAGE.

To purify the target protein, the fermentation supernatant was filtered by $0.45 \mu \mathrm{m}$ filter membrane, and loaded into a $5 \mathrm{~mL}$ His-Trap column pre-equilibrated with buffer A (25 mM Tris- $\mathrm{HCl}, 150 \mathrm{mM}$ sodium chloride, $\mathrm{pH}$ 7.5). Then washed by buffer B (buffer A with $10 \mathrm{mM}$ imidazole), the target protein was eluted with buffer C (buffer A with $200 \mathrm{mM}$ imidazole). Then eluent fractions containing the target protein were concentrated using Amicon Ultracentricon (Millipore) with a $30 \mathrm{kDa}$ cutoff, and desalted via GE HiTrap desalting column to exclude imidazole. The purified BINagZ were detected by SDS-PAGE. The 
concentration of protein was determined by the Bradford assay using bovine serum albumin (BSA) as the standard.

\section{Enzyme Assays}

The activity of purified BlNagZ was determined by measuring the hydrolysis of pNP- $\beta$-D-GlcNAc (Yem and Wu, 1976), which was dissolved in $50 \mathrm{mM}$ phosphate buffer ( $\mathrm{pH}$ 5.5) solution at a concentration of $1 \mathrm{mM}$. In the reaction, $10 \mu \mathrm{L}$ of protein fraction was mixed with $90 \mu \mathrm{L}$ of $1 \mathrm{mM}$ pNP- $\beta$-D-GlcNAc and incubated at $60^{\circ} \mathrm{C}$ for $10 \mathrm{~min}$. The reaction was quenched with $200 \mu \mathrm{L}$ of $200 \mathrm{mM}$ sodium carbonate. The activity was determined in 96 well plates by measuring the absorption at $405 \mathrm{~nm}$ of p-Nitrophenol. One unit of activity is the amount of enzyme that released $1 \mu \mathrm{mol}$ p-Nitrophenol per min at appropriate conditions.

\section{Effects of pH and Temperature on the Enzyme Activity and Stability}

The optional $\mathrm{pH}$ of BlNagZ was determined in various $50 \mathrm{mM}$ buffers over a $\mathrm{pH}$ range from 4.0 to 8.0 . To analyze the $\mathrm{pH}$ stability, the enzyme samples were incubated in $50 \mathrm{mM}$ buffers with different $\mathrm{pH}$ at $4^{\circ} \mathrm{C}$ for $12 \mathrm{~h}$ and the residual activity was assayed by the standard method.

The effects of temperature on the enzyme activity were determined at different temperatures ranging from 30 to $90^{\circ} \mathrm{C}$ in $50 \mathrm{mM}$ sodium phosphate buffer ( $\mathrm{pH}$ 5.5). The thermostability was tested by pre-incubating BlNagZ at 55,60 , and $65^{\circ} \mathrm{C}$ in $50 \mathrm{mM}$ sodium phosphate buffer $(\mathrm{pH} 5.5)$ for various times, respectively, and measuring the residual activity.

\section{Effects of Metal lons and Organic Reagents on Enzyme Activity}

Different metal ions were added to the reaction mixture at a $5 \mathrm{mM}$ final concentration to test their effects on the enzyme activity of BlNagZ. The effects of $5 \%$ organic reagents on the activity of BlNagZ were determined under optimal conditions ( $\mathrm{pH} 5.5$, $60^{\circ} \mathrm{C}$ ). The reaction was performed at $60^{\circ} \mathrm{C}$ for $10 \mathrm{~min}$. The activity of the enzyme without any additions was set as $100 \%$.

\section{Optimization of Induction Expression and Secretion Conditions}

There are many factors that affect the expression and secretion of BlNagZ. To increase the secretion of BlNagZ, the fermentation conditions were optimized. First, to investigate the effect of IPTG concentration on protein expression, IPTG with different concentration of $0.5,0.75,1.0$, and $1.25 \mathrm{mM}$ was used to induce at $37^{\circ} \mathrm{C}$. Then, to investigate the effect of temperature on protein expression, the induction was performed at different temperatures of $18,25,30$, and $37^{\circ} \mathrm{C}$ after the addition of $1 \mathrm{mM}$ IPTG. Last, the effect of the different cell density on protein expression was researched, different initial induced cell density of $O D_{600} 1.0,1.5,2.0,2.5,3.0$, and 3.5 was performed to induced by $1 \mathrm{mM}$ IPTG at $25^{\circ} \mathrm{C}$.

Finally, all the factors including temperature $(20,25,30$, and $\left.37^{\circ} \mathrm{C}\right)$, IPTG concentration $(0.5,0.75,1.0$, and $1.25 \mathrm{mM})$ and initial induced cell density of $\operatorname{OD}_{600}(2.0,2.5,3.0$, and
3.5) were designed by software Orthogonal Design Assistant (Supplementary Table 1). The $O D_{600}$ and enzyme activity of all the above samples were detected per $4 \mathrm{~h}$ after induction.

\section{Combinatory Hydrolysis of Chitin}

To study the hydrolytic activity of BlNagZ, firstly, $\mathrm{N}, \mathrm{N}^{\prime}$ Diacetylchitobiose was used as the substrate. The $500 \mu \mathrm{L}$ reaction system (50 mM sodium phosphate buffer, pH 5.5), including 450 $\mu \mathrm{L}$ of $1 \mathrm{mM} \mathrm{N}, \mathrm{N}^{\prime}$-Diacetylchitobiose and $50 \mu \mathrm{L}$ enzyme (13.5 $\mathrm{U} / \mathrm{mL}$ ) was incubated at $55^{\circ} \mathrm{C}$ for $15 \mathrm{~min}$. Then the mixture was filtered by $0.22 \mu \mathrm{m}$ filter membrane after processed at $100^{\circ} \mathrm{C}$ for $10 \mathrm{~min}$. The end products were analyzed by HPLC (Du et al., 2019).

Then the colloidal chitin was used as the substrate. The 500 $\mu \mathrm{L}$ reaction system (50 mM sodium phosphate buffer, $\mathrm{pH} 5.5$ ), including $300 \mu \mathrm{L}$ of $3 \%$ colloidal chitin and $200 \mu \mathrm{L}$ enzyme (13.5 $\mathrm{U} / \mathrm{mL}$ ) was incubated at $55^{\circ} \mathrm{C}$ for $4 \mathrm{~h}$. Then the products were analyzed by HPLC.

To increase the production of GlcNAc, the chitinase ChiA was used to hydrolyze colloidal chitin with BlNagZ. In the reaction system (50 mM sodium phosphate buffer, pH 5.5), $200 \mu \mathrm{L}$ ChiA $(15 \mathrm{U} / \mathrm{mL})$ was added into the $400 \mu \mathrm{L}$ of $30 \%$ colloidal chitin, and the mixture was incubated at $50^{\circ} \mathrm{C}$ for $3 \mathrm{~h}$. Then, different amounts of $\mathrm{BlNagZ}(0,0.65,1.3,1.95,2.6 \mathrm{U}$, respectively) were added into the mixture, the total reaction was put to a constant volume of $1 \mathrm{~mL}$ using the same buffer. The products were analyzed by $\mathrm{HPLC}$ after $55^{\circ} \mathrm{C}$ incubation for $20 \mathrm{~min}$.

\section{RESULTS}

\section{Gene Cloning and Sequence Analysis}

The amino acid sequence of BsNagZ (GenBank: CAB11942.1) was subjected to protein BLAST in the NCBI database to search for new NAGases. Several new NAGases that show 30-70\% amino acid similarity with BsNagZ were selected for heterologous expression and characterization, including BlNagZ of B. licheniformis WX-02. BlNagZ (GenBank: CP012110.1) showed $68.06 \%$ sequence identity to BsNagZ, which consists of 619 amino acids with a signal peptide located at 1st-26th aa of $\mathrm{N}$ terminal $^{2}$. The theoretical molecular mass and pI of BlNagZ were $67.92 \mathrm{kDa}$ and 8.75 , respectively.

Through literature review, we found that 4 other NAGases with homology to BlNagZ have been studied in detail besides BsNagZ. These four NAGases are BpNagZ from Bacillus pumilus (GenBank: MK559425), NagZ703 from Bacillus pseudofirmus OF4 (GenBank: ADC51622.1), Cht60 from Alteromonas sp. strain O-7 (GenBank: D17399.1), and NagA from Streptomyces thermoviolaceus (GenBank: AB008771.1), which showed 67.26, $39.2,33.81$, and $31.2 \%$ sequence identity to BlNagZ, respectively. The amino acid sequence alignment between BlNagZ and the other NAGases was shown in Figure 1. The highly conserved sequence segment KH(F/I)PG(H/L)GX(4)D(S/T)H of GH3 family (Litzinger et al., 2010) was found in BlNagZ from

\footnotetext{
${ }^{2}$ http://www.cbs.dtu.dk/services/signalp
} 

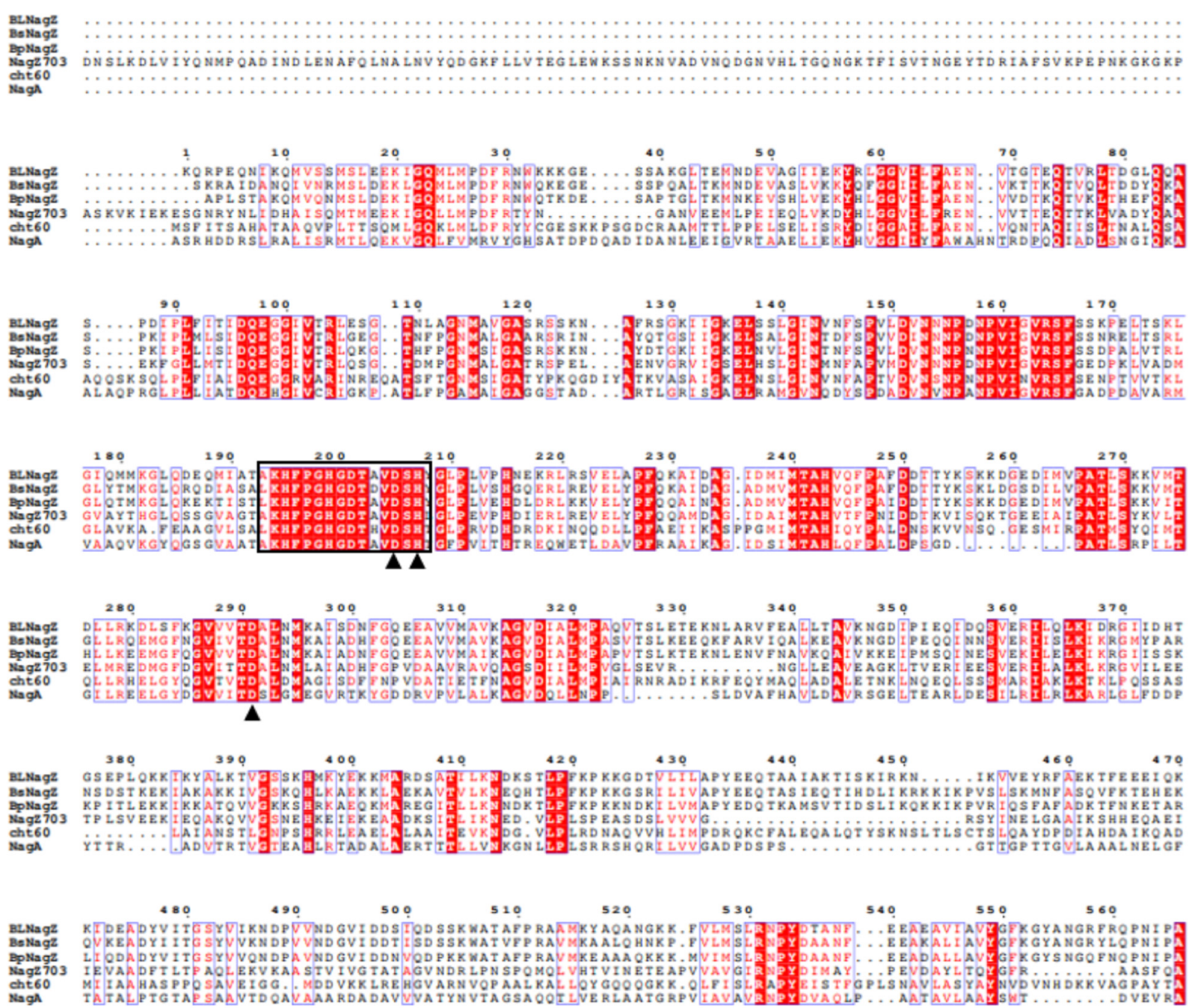

che60

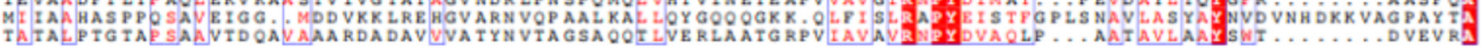

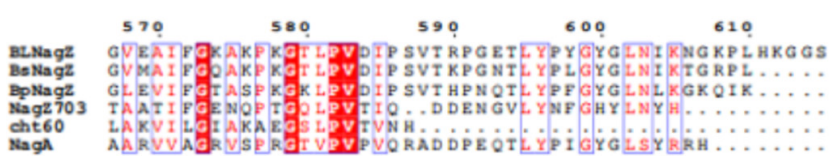

FIGURE 1 | Multiple sequences alignments of BINagZ with reported GH3 NAGases. Strictly conserved residues are indicated in white letters on a red background, and conservatively substituted residues are boxed. The catalytic active sites (D205, H207, D291) are indicated by solid triangle "\$". The highly conserved sequence segment $\mathrm{KH}(\mathrm{F} / \mathrm{I}) \mathrm{PG}(\mathrm{H} / \mathrm{L}) \mathrm{GX}(4) \mathrm{D}(\mathrm{S} / \mathrm{T}) \mathrm{H}$ of $\mathrm{GH} 3$ family NAGase are Black box.

$\mathrm{K}^{194}$ to $\mathrm{H}^{207}$, and the typical catalytic activity sites D205H207-D291 was also found, which suggested BlNagZ was a $\mathrm{N}$-acetylglucosaminidase of GH3 family.

\section{Expression and Purification of Recombinant BINagZ}

The recombinant BlNagZ was expressed soluble in E. coli BL21 (DE3) without obvious inclusion body. The SDS-PAGE analysis showed BlNagZ could secret into the medium and the purified BlNagZ migrated as a single band with a molecular mass of about $70 \mathrm{kDa}$, which was in accordance to the theoretically calculated mass (Figure 2). The pelB signal peptide was excised from BlNagZ when it was secreted. The specific activity of the enzyme after each purification step was calculated (Table 1). The results showed that the enzyme was stable during the steps of purification. The BlNagZ activity in the fermentation supernatant was $3.2 \mathrm{U} / \mathrm{mL}$, and the specific activity reached $13.05 \mathrm{U} / \mathrm{mg}$ after purification.

\section{Biochemical Characterization of BINagZ}

The activity and stability of purified $\mathrm{BlNagZ}$ were assayed at a temperature range from 30 to $90^{\circ} \mathrm{C}$ and $\mathrm{pH}$ range from 3.5 to 8 . The optimum temperature is $60^{\circ} \mathrm{C}$ (Figure $3 \mathrm{~A}$ ). BlNagZ shows poor thermal stability at $60^{\circ} \mathrm{C}$, only $50 \%$ of the residual activity 


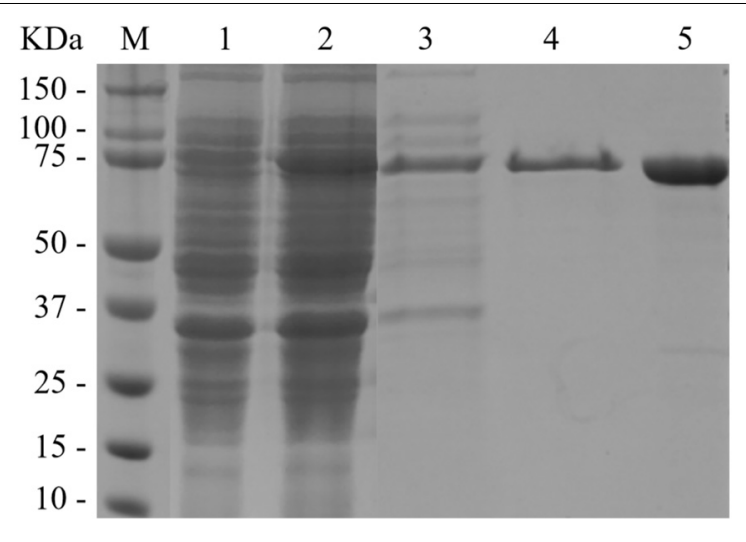

FIGURE 2 | SDS-PAGE analysis of BINagZ at each purification step. Lane M, prestained protein ladder; Lane 1, Bacterial precipitation of induced $E$. coli BL21 (DE3)-pET26b-BINagZ cells; Lane 2, cell extract of induced E. coli BL21 (DE3)-pET26b-BINagZ cells; Lane 3, fermentation supernatant of induced E. coli BL21 (DE3)-pET26b-BINagZ cells; Lane 4, Purification on Ni-NTA affinity column; Lane 5, Purification on desalting column.

TABLE 1 | Purification steps of recombinant BINagZ.

\begin{tabular}{lcccc}
\hline Purification & $\begin{array}{c}\text { Total protein } \\
\text { (mg) }\end{array}$ & $\begin{array}{c}\text { Total } \\
\text { activity (U) }\end{array}$ & $\begin{array}{c}\text { Specific activity } \\
\text { (U/mg) }\end{array}$ & $\begin{array}{c}\text { Purification } \\
\text { (-fold) }\end{array}$ \\
\hline Crude enzyme & 120 & 1073 & 8.95 & 1 \\
Ni-column & 20.09 & 244.69 & 12.18 & 1.36 \\
Desalting column & 7.03 & 91.81 & 13.05 & 1.46 \\
\hline
\end{tabular}

remains after at $60^{\circ} \mathrm{C}$ for $30 \mathrm{~min}$, and it keeps stable at $55^{\circ} \mathrm{C}$ with $90 \%$ residual activity after $30 \mathrm{~min}$ treatment (Figure 3B). The optimum $\mathrm{pH}$ of $\mathrm{BlNagZ}$ is 5.5 , and it presents over $70 \%$ relative activity between $\mathrm{pH} 5$ and 7 (Figure 3C). BlNagZ can retain over $70 \%$ residual activity between $\mathrm{pH} 4$ and 6.5 after incubation at $4^{\circ} \mathrm{C}$ overnight (Figure 3D), which shows BlNagZ is stable in weak acidic conditions.

The effects of metal ions on the activity of BlNagZ toward pNP- $\beta$-GlcNAc were shown in Table $2 . \mathrm{K}^{+}, \mathrm{Na}^{+}, \mathrm{Li}^{+}, \mathrm{Mg}^{2+}$, and $\mathrm{Mn}^{2+}$ have no obvious effects on the activity of BlNagZ. But $\mathrm{Cu}^{2+}$ and $\mathrm{Hg}^{2+}$ almost abolish the enzymatic activity since $\mathrm{Hg}^{2+}$ and $\mathrm{Cu}^{2+}$ can act with free carboxyl groups in protein and form insoluble salts to inhibit the activity of most enzymes (Jorobekova et al., 2005). The addition of $5 \mathrm{mM}$ EDTA didn't affect the activity of BlNagZ, which suggested that BlNagZ isn't a metaldependent enzyme. Organic solvents such as methanol, alcohol and isopropanol showed slight inhibition, while formaldehyde inhibited most of the activity, because formaldehyde can denature enzyme by interacting with hydroxyl, sulfhydryl or amino groups of enzymes (Kamps et al., 2019). Glycerol and DSMO didn't change the activity, which indicated that BlNagZ has better tolerance to organic solvents.

\section{The Culture Condition Optimization to Improve the Secretion of BINagZ}

The recombinant strain BL21 (DE3)-pET26b-BlNagZ can express and secret BlNagZ into the medium, and the BlNagZ's activity reached $3.2 \mathrm{U} / \mathrm{mL}$ when induced at $37^{\circ} \mathrm{C}$ with $0.5 \mathrm{mM}$ IPTG for $32 \mathrm{~h}$ and initial $O D_{600}$ of 2, respectively. In order to improve the extracellular yield of BlNagZ, we optimized the expression parameters, such as the concentration of IPTG, inducted temperature, and time, etc.

\section{Effects of IPTG on the Secretion of BINagZ}

By contrast, dose-dependent expression when using IPTG as an inducer is not possible since IPTG can enter the cell by active transport through the Lac permease or by permease independent pathways. Since the expression of Lac permease is heterogeneous and the number of active permeases in each cell is highly variable, protein expression does not respond predictably to IPTG concentration (Rosano and Ceccarelli, 2014). Different amounts of IPTG were added into the culture, respectively, when the $O D_{600}$ reached 2.0 (Figure 4A). Compared to the control, the expression level of BlNagZ in the medium increased along with the increase of IPTG concentration. When the concentration of IPTG increased to $1.0 \mathrm{mM}$, the BlNagZ activity in the fermentation supernatant reached the highest of $5.3 \mathrm{U} / \mathrm{mL}$ at $36 \mathrm{~h}$. Then its activity decreased when IPTG concentration increased to $1.25 \mathrm{mM}$. Meanwhile, different concentrations of IPTG has no obvious effect on cell growth. Therefore, $1.0 \mathrm{mM}$ IPTG was more conducive to the secretion expression of BlNagZ.

\section{Effects of Temperature on the Secretion of BINagZ}

Temperature is also an important factor for recombinant protein production in E. coli (Fang et al., 2011). In general, E. coli could slow down the synthesis rate of recombinant proteins when growing at a lower temperature, thereby increasing its solubility. However, it would lead to lower protein yield. To optimize the temperature for the production of BlNagZ, the strain was induced by $1 \mathrm{mM}$ IPTG at $20,25,30$, and $37^{\circ} \mathrm{C}$, respectively. The results showed that the activity of BlNagZ in fermentation supernatant was highest of $11.2 \mathrm{U} / \mathrm{mL}$ when induced at $25^{\circ} \mathrm{C}$ for $40 \mathrm{~h}$, which was 1.1 higher than induced at $30^{\circ} \mathrm{C}$, and 1.7 higher than induced at $37^{\circ} \mathrm{C}$ (Figure $4 \mathrm{~B}$ ). In comparison, BlNagZ was hardly secreted outside the cells at $20^{\circ} \mathrm{C}$. Induction at $25^{\circ} \mathrm{C}$ led to better coordination between the protein synthesis and cell growth. There was little difference in cell density while the strain was cultured under 25,30 , and $37^{\circ} \mathrm{C}$, respectively. Therefore, $25^{\circ} \mathrm{C}$ was the best-induced temperature.

\section{Effects of the Initial Induced Cell Density on the Secretion of BINagZ}

We then optimized the initial induced cell density at the condition of $1 \mathrm{mM}$ IPTG and $25^{\circ} \mathrm{C}$. The results showed that the activity of BlNagZ in the supernatant increased with the increase of starting cell density, and up to the highest $13 \mathrm{U} / \mathrm{mL}$ at 3 of $O D_{600}$ after induction of $42 \mathrm{~h}$ (Figure 4C). Within the initial induced $O D_{600}$ range of 2.5-3.5, the secretion expression levels of $\mathrm{BlNagZ}$ were similar. It is feasible for induction when the initial $O D_{600}$ is at the range from 2.5 to 3.5 .

\section{Optimization of Induction Expression Conditions by Orthogonal Experiments}

According to the results of orthogonal experiments, temperature is the most important factor affecting the expression and 

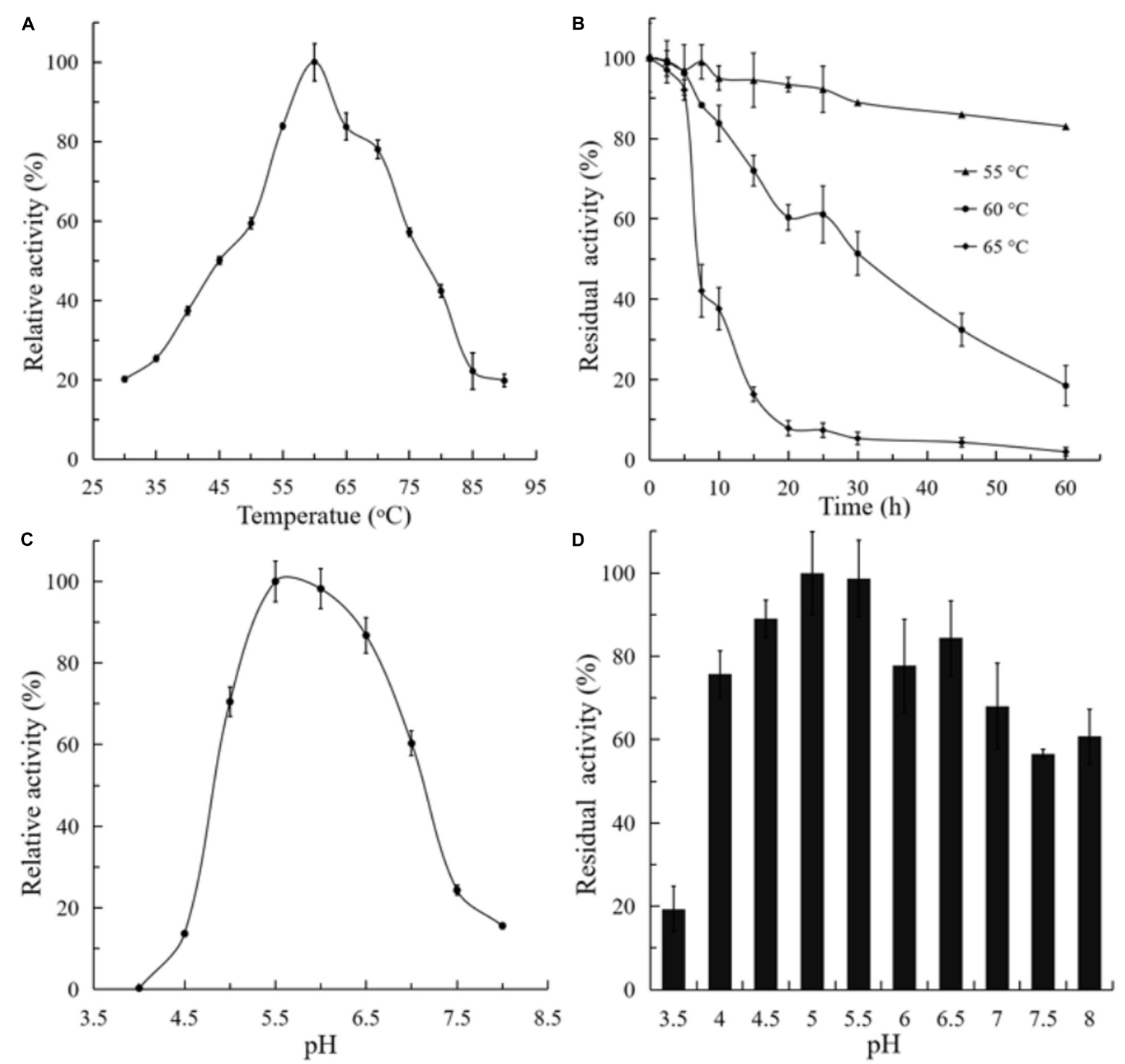

FIGURE 3 | Effects of temperature and pH on recombinant BINagZ activity. (A) Effects of temperature on the activity of BINagZ. The reaction was conducted from 30 to $90^{\circ} \mathrm{C}$ in $50 \mathrm{mM}$ phosphate buffer ( $\mathrm{pH}$ 5.5); (B) effects of temperature on the stability of BINagZ. The BINagZ was pre-incubated at the indicated temperature in $50 \mathrm{mM}$ phosphate buffer ( $\mathrm{pH} 5.5)$ for $30 \mathrm{~min}$. After pre-incubation, the residual activity of BINagZ was measured at pH 5.5 and $60^{\circ} \mathrm{C}$. (C) Effects of pH on the activity of BINagZ. The buffers used were $50 \mathrm{mM}$ citric phosphate $(\mathrm{pH} 4-8)$ at $60^{\circ} \mathrm{C}$. (D) Effects of $\mathrm{pH}$ on the stability of BINagZ. The recombinant enzyme was incubated at different $\mathrm{pH}$ for $12 \mathrm{~h}$ at $4^{\circ} \mathrm{C}$. After incubation, the residual activity of BINagZ was measured at $\mathrm{pH} 5.5$ and $60^{\circ} \mathrm{C}$. Each value of the assay was the arithmetic mean of triplicate measurements.

secretion of BlNagZ while the initial induced $O D_{600}$ had the minimal effect (Supplementary Table 1). In all, $1 \mathrm{mM}$ IPTG induction at $25^{\circ} \mathrm{C}$ for $40 \mathrm{~h}$ with initial $O D_{600} 3.5$ would be the best fermentation condition for secretion of BlNagZ and its activity can increase to $13.62 \mathrm{U} / \mathrm{mL}$ from $3.2 \mathrm{U} / \mathrm{mL}$ (Figure 4D).

\section{Hydrolytic Colloid Chitin to Produce GIcNAc}

Firstly, N, N'-Diacetylchitobiose and colloidal chitin were used as substrates to verify the hydrolytic activity of BINagZ. The results showed that BlNagZ could hydrolyze N, N'-Diacetylchitobiose into GlcNAc completely in 15 min (Figures 5A-C). However, the efficiency of this enzyme to directly hydrolyze colloidal chitin is very low, only a small amount of GlcNAc was produced (Figure 5D), which was consistent with previous reports (Jiang et al., 2019).

To increase the output of GlcNAc, 30\% colloidal chitin was firstly hydrolyzed with chitinase ChiA (Song et al., 2020) for $3 \mathrm{~h}$, and then hydrolyzed with different amount of BlNagZ for $30 \mathrm{~min}$. The results showed that BlNagZ can effectively hydrolyze $\mathrm{N}, \mathrm{N}^{\prime}$-Diacetylchitobiose produced by ChiA into GlcNAc, and 2.6 $\mathrm{U} / \mathrm{ml}$ of BlNagZ could convert the hydrolysis product of ChiA into GlcNAc completely. Thus, the yield ratio of GlcNAc from 
TABLE 2 | Effects of metal ions and chemical reagents on BINagZ activity.

\begin{tabular}{|c|c|c|c|c|c|}
\hline Metal ions or chemicals & Concentration & Relative activity (\%) & Metal ions or chemicals & Concentration & Relative activity (\%) \\
\hline No addition & 0 & $100 \pm 2.61$ & $\mathrm{Hg}^{2+}\left(\mathrm{HgCl}_{2}\right)$ & $5 \mathrm{mM}$ & $0.026 \pm 0.10$ \\
\hline $\mathrm{Li}^{+}(\mathrm{LiCl})$ & $5 \mathrm{mM}$ & $95.12 \pm 2.08$ & EDTA & $5 \mathrm{mM}$ & $88.43 \pm 1.65$ \\
\hline $\mathrm{K}^{+}(\mathrm{KCl})$ & $5 \mathrm{mM}$ & $92.04 \pm 8.54$ & Methanol & $5 \%$ & $90.76 \pm 0.50$ \\
\hline $\mathrm{Na}^{+}(\mathrm{NaCl})$ & $5 \mathrm{mM}$ & $106.22 \pm 7.27$ & Alcohol & $5 \%$ & $71.18 \pm 0.18$ \\
\hline $\mathrm{Mg}^{2+}\left(\mathrm{MgCl}_{2}\right)$ & $5 \mathrm{mM}$ & $94.28 \pm 4.88$ & Isopropanol & $5 \%$ & $74.84 \pm 0.19$ \\
\hline $\mathrm{Mn}^{2+}\left(\mathrm{MnCl}_{2}\right)$ & $5 \mathrm{mM}$ & $96.45 \pm 0.42$ & Glycerol & $5 \%$ & $100.4 \pm 0.21$ \\
\hline $\mathrm{Ca}^{2+}\left(\mathrm{CaCl}_{2}\right)$ & $5 \mathrm{mM}$ & $78.85 \pm 2.94$ & Formaldehyde & $5 \%$ & $7.47 \pm 0.15$ \\
\hline $\mathrm{Cu}^{2+}\left(\mathrm{CuCl}_{2}\right)$ & $5 \mathrm{mM}$ & $0.51 \pm 0.41$ & DSMO & $5 \%$ & $100.2 \pm 0.32$ \\
\hline
\end{tabular}

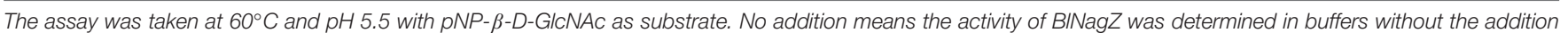
of any ions or chemicals. Each value of the assay was the arithmetic mean of triplicate measurements.

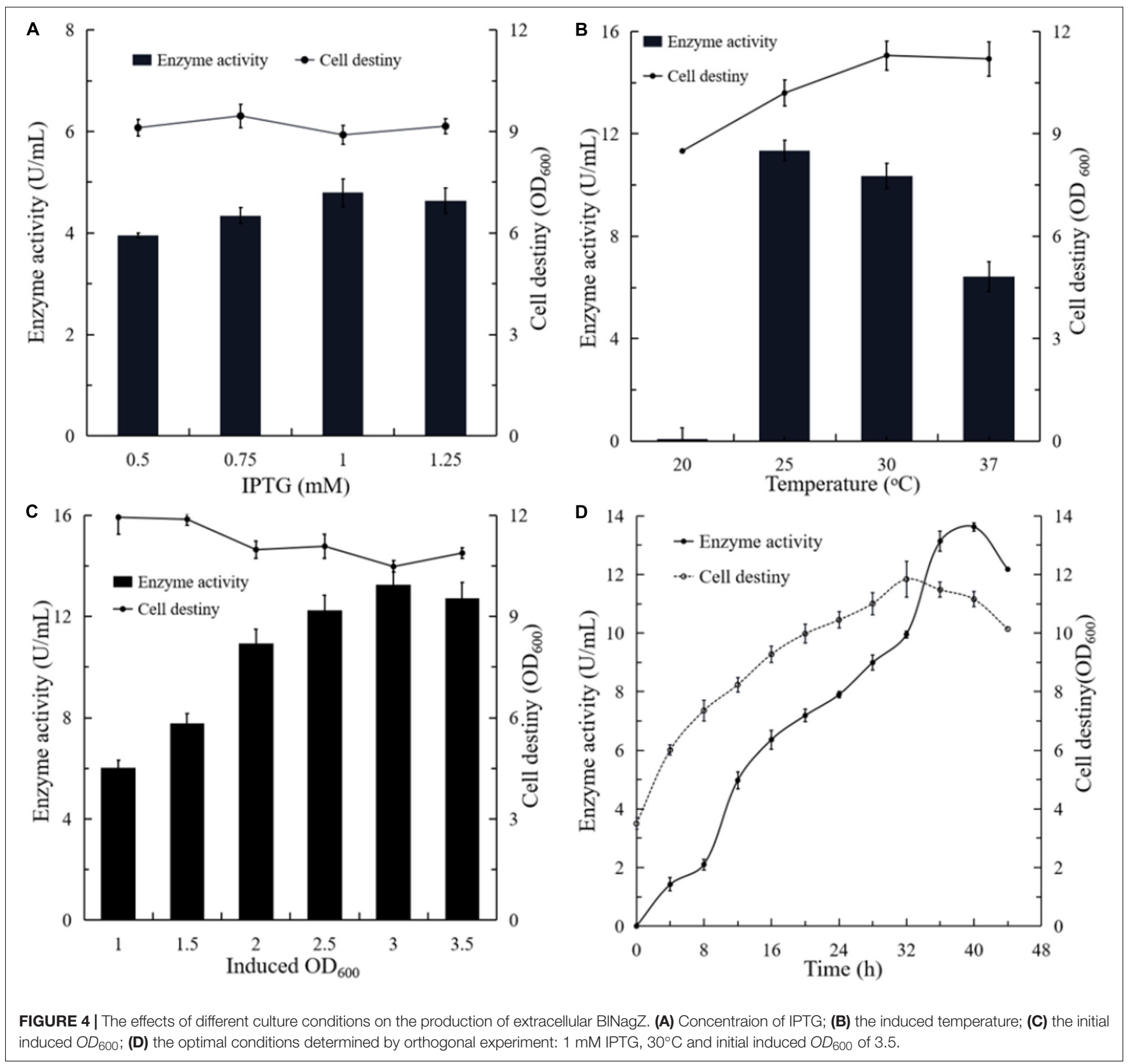




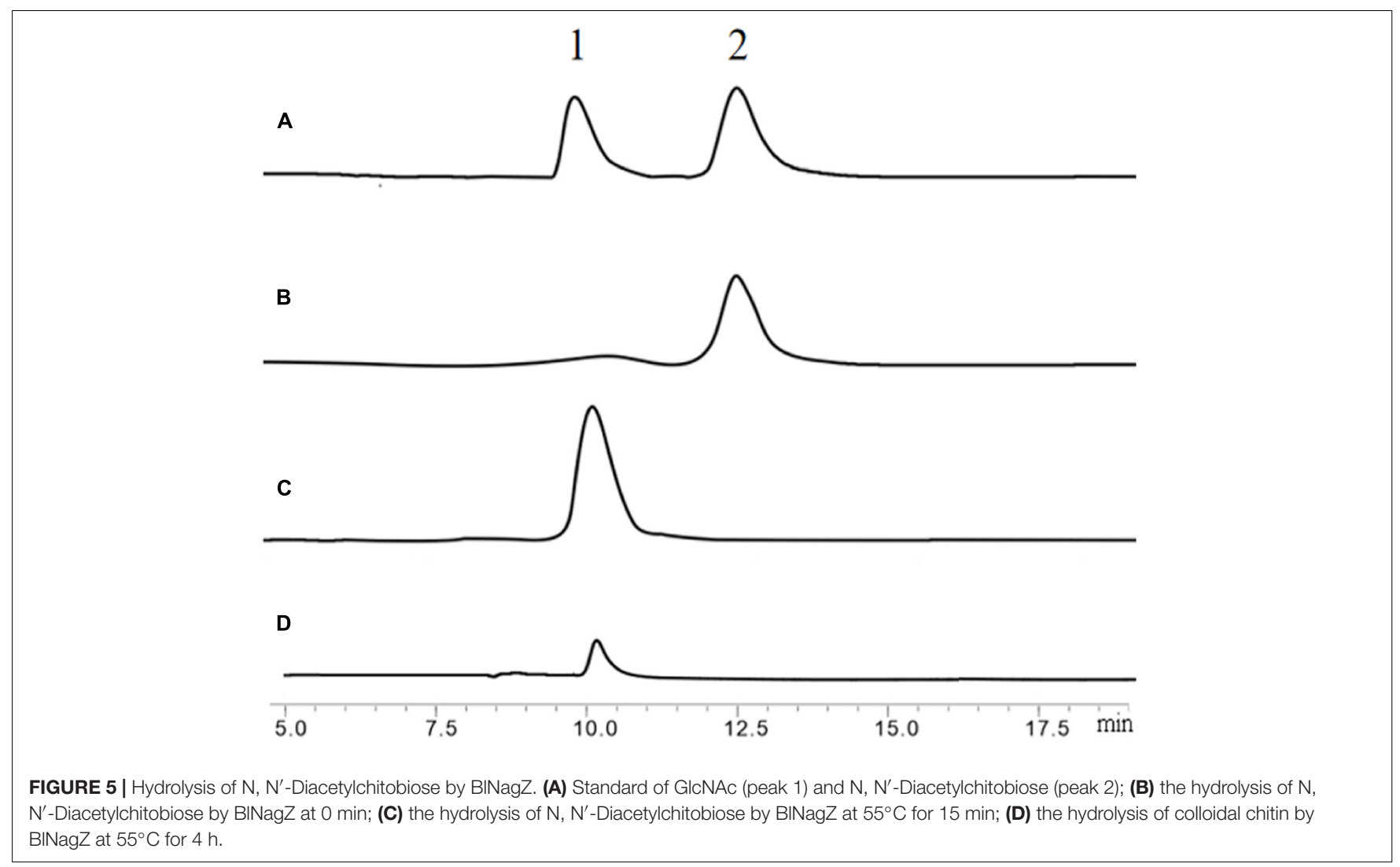

$30 \%$ colloidal chitin could reach $89.2 \%$ within 3.5 h using the combination of ChiA and BlNagZ (Figure 6).

\section{DISCUSSION}

In this study, BlNagZ gene obtained from B. licheniformis WX-02 was expressed extracellularly in E. coli with PelB signal peptide. BlNagZ contained highly conserved sequence $\mathrm{KH}(\mathrm{F} / \mathrm{I}) \mathrm{PG}(\mathrm{H} / \mathrm{L}) \mathrm{GX}(4) \mathrm{D}(\mathrm{S} / \mathrm{T}) \mathrm{H}$ and the catalytic active center "D-H-D" that were typical in NAGases of GH 3 family. It can hydrolyze $\mathrm{N}, \mathrm{N}^{\prime}$-Diacetylchitobiose and colloidal chitin to produce GlcNAc. These results demonstrated that BlNagZ was a NAGase of GH3 family.

Enzyme activity and stability, especially thermal and $\mathrm{pH}$ stability, must be considered in the industrial application. A few NAGases have good stability at $60^{\circ} \mathrm{C}$ or above, such as BsNagZ (Jiang et al., 2019), BpNagZ from B. pumilus (Du et al., 2019), CbsA from Thermotoga neapolitana (Kim et al., 2015), and NahA from Symbiobacterium thermophilum (Ogawa et al., 2006; Table 3). In this research, the optimum reaction temperature and $\mathrm{pH}$ of $\mathrm{BlNagZ}$ was $60^{\circ} \mathrm{C}$ and 5.5. And BlNagZ showed great thermostability, which remained 90 and $50 \%$ residual activity after pre-incubation at 55 and $60^{\circ} \mathrm{C}$ for $30 \mathrm{~min}$, respectively. Similarly, BlNagZ had high activity over $\mathrm{pH}$ range from 5.5 to 6.5 , and remained stable from $\mathrm{pH} 4.5$ to 6.5 . Acid-activity and acid-stability are desirable properties for enzymatic hydrolysis of chitin in an acidic environment. Therefore, BlNagZ is suitable to degrade chitin to GlcNAc in industry, and more importantly, its activity is the highest among the reported acid-stable NAGase.

Conventional expression of heterologous protein inside E. coli is complicated for industrial applications for cell disruption and protein purification. Here, BlNagZ was guided to the periplasmic space via PelB signal peptide with the property of periplasmic targeting, then secreted into the medium. According to Huang's study, temperature, IPTG concentration, and induction time were determinant factors for the expression and secretion of CSN mediated by PelB (Huang et al., 2016). Therefore, we optimized the IPTG concentration, temperature, initial $O D_{600}$ and induced time. BlNagZ activity of the supernatant increased to $13.62 \mathrm{U} / \mathrm{mL}$, the expression of BlNagZ was almost $1.04 \mathrm{mg} / \mathrm{mL}$, which was 4.25 times higher than optimization before (Figure 4) and about $70 \%$ of the BlNagZ can secret outside the cells. In other studies on the secretion expression of NAGases, only BsNagZ was secreted and expressed in $P$. pastoris, and the protein expression level reached $1.10 \mathrm{mg} / \mathrm{mL}$ at the 14th day (Jiang et al., 2019). Both of which have similar expression levels, but BlNagZ has higher activity and the fermentation time in $E$. coli is much shorter. There are also some proteins extracellular expressed in E. coli by PelB signal peptide. Such as Candida antarctica lipase B in the culture supernatant amounted to $0.55 \mathrm{mg} / \mathrm{mL}$ under the optimized conditions (Ujiie et al., 2016); The extracellular xylanase reached $1 \mathrm{mg} / \mathrm{mL}$ (Chang et al., 2017). Secretory expression greatly simplifies the process of protein purification. Moreover, the increase of the expression level reduces the production cost of the enzyme. Both are conducive to industrial application. 
TABLE 3 | Biochemical characterization of the characterized GH3 NAGases.

\begin{tabular}{|c|c|c|c|c|c|c|c|}
\hline Protein & Source & $\begin{array}{l}\text { Optimal } \\
\text { pH }\end{array}$ & $\begin{array}{c}\text { Optimal } \\
\text { temperature }\left({ }^{\circ} \mathrm{C}\right)\end{array}$ & $\begin{array}{c}\text { Specific activity } \\
\text { (U/mg) }\end{array}$ & $\begin{array}{l}K_{m}(\mu \\
\mathrm{mol} / \mathrm{L})\end{array}$ & $\begin{array}{l}V_{\max }(\mu \\
\mathrm{mol} / \mathrm{min})\end{array}$ & $\begin{array}{l}\text { Half-life } \\
\left(\min ,{ }^{\circ} \mathrm{C}\right)\end{array}$ \\
\hline BINagZ & B. licheniformis & 5.5 & 60 & 13.05 & 932 & 43.7 & 30,60 \\
\hline BsNagZ & B. subtilis (Jiang et al., 2019) & 6.0 & 60 & 2.9 & 1,130 & 39.27 & 30,60 \\
\hline BpNagZ & B. pumilus (Du et al., 2019) & 6.0 & 70 & 5.91 & 90.74 & 136.02 & 20,70 \\
\hline CbsA & Thermotoga neapolitana (Kim et al., 2015) & 8.0 & 75 & - & - & 0.3 & - \\
\hline NahA & Symbiobacterium thermophilum (Ogawa et al., 2006) & 5.5 & 65 & 3.05 & - & - & 30,60 \\
\hline NagA & Streptomyces thermoviolaceus (Tsujibo et al., 1998) & 5.0 & 60 & 64.4 & 425.7 & 24.8 & - \\
\hline $\operatorname{Nag} A$ & Pseudomonas fluorescens (Park et al., 2015) & 8.0 & 37 & 5.3 & 130 & 245 & 60,50 \\
\hline NagA & Trichinella spiralis (Bruce and Gounaris, 2006) & 4.4 & 54 & 0.03 & 187 & - & 50,54 \\
\hline rNag3HWLB1 & Sphingobacterium sp. HWLB1 (Zhou et al., 2016) & 7.0 & 40 & - & 1,120 & 19.88 & 15,50 \\
\hline
\end{tabular}

"-" means the data haven't been provided.

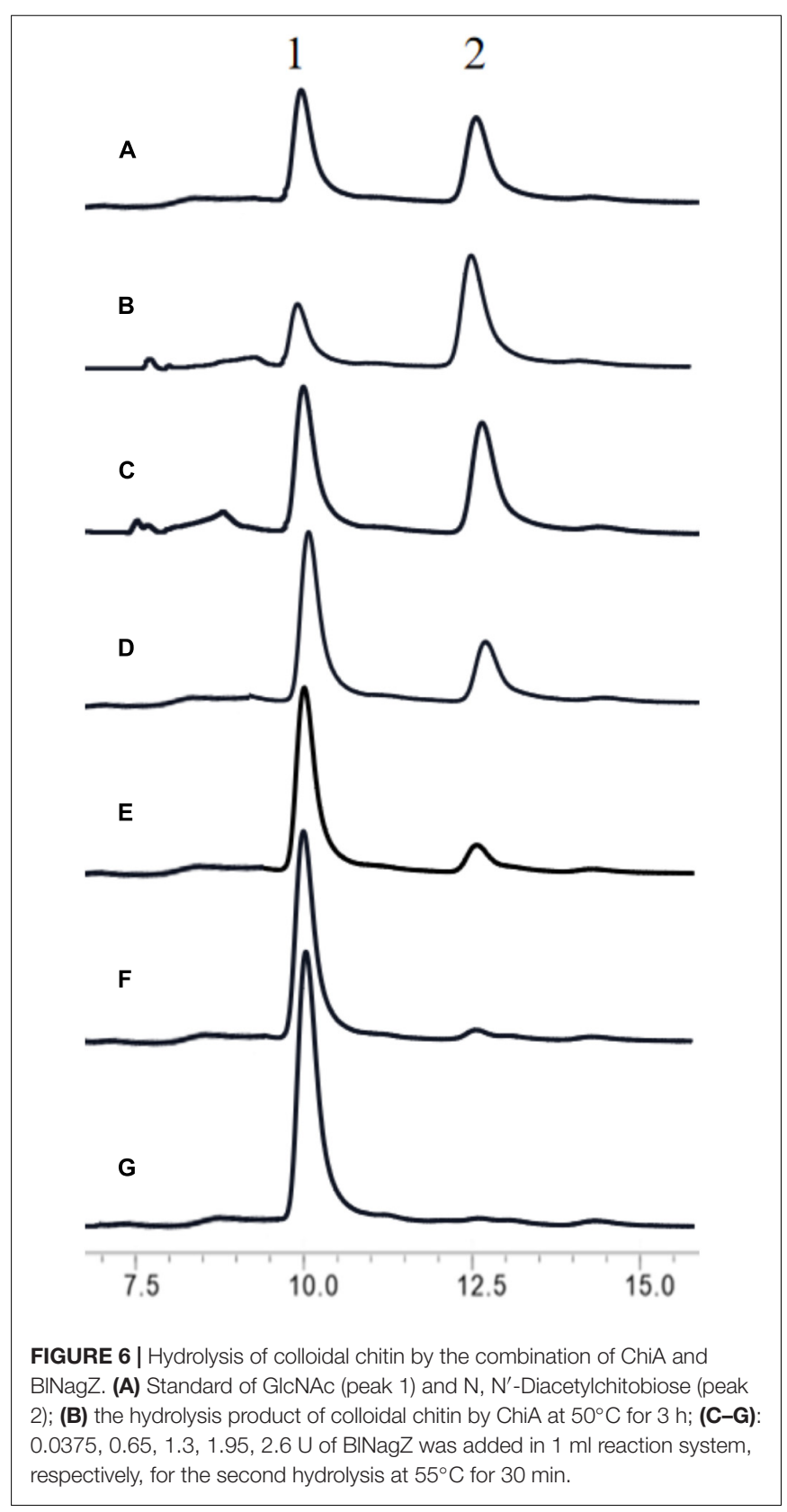

To our knowledge, NAGases are very inefficient at digesting chitin because of their cutting mode (Litzinger et al., 2010). And most of chitinases can not hydrolyze chitin into GlcNAc completely. Therefore, the high efficient conversion of chitin into GlcNAc requires the combinatory action of chitinase and NAGase (Nguyen-Thi and Doucet, 2016). For example, Fu et al. (2014) reported that NAGases from Streptomyces coelicolor combined with chitinase C could produce $27.8 \mathrm{mg} / \mathrm{mL}$ GlcNAc with a conversion rate of $92.6 \%$ after $24 \mathrm{~h}$ digestion. In Zhou et al.'s (2016) work, a small amount of GlcNAc was obtained by hydrolysis of chitin with Chitinase CtnSg, however, the yield of GlcNAc enhanced 3.74-fold by the synergy of CtnSg and GlcNAcase rNag3HWLB1. Wang et al. (2018) used BsChi from B. subtilis and OfHex1, a NAGase from the insect Ostrinia furnacalis to produce GlcNAc with 95\% purity from pretreated crab shells with a yield rate of $60 \%$. In our research, BlNagZ has the ability to hydrolyze chitin and produce GlcNAc with very low efficiency (Figure 5D). Combining with chitinase ChiA from B. licheniformis (Song et al., 2020), the yield rate of GlcNAc increased to $89.2 \%$. This is slightly less than Fu et al.'s (2014) 92.6\% and Wang et al.'s (2018) 95\% conversion ratio. But the reaction time greatly reduced from more than 24 to $3.5 \mathrm{~h}$ by using enzymes with higher activities, which is also far lower than our previously reported $12 \mathrm{~h}$ (Song et al., 2020).

Usually, high substrate concentration would inhibit the enzyme activity. While in industry application, a high concentration of substrate can decrease the reaction volume, and then lower the production cost. It was found that ChiA can act on $30 \%(\mathrm{w} / \mathrm{v})$ colloidal chitin with a high conversion ratio (Song et al., 2020). In this study, ChiA and BlNagZ are also combined to hydrolyze $30 \%$ colloidal chitin. Since chiA can efficiently hydrolyze chitin into (GlcNAc) $)_{2}$. Then BlNagZ can efficiently hydrolyze (GlcNAc) 2 to GlcNAc. This greatly shortens the hydrolysis time by taking full advantage of these two enzymes. After hydrolysis by ChiA for $3 \mathrm{~h}$, the substrate was then digested by BINagZ for another $30 \mathrm{~min}$, the conversion ratio of GlcNAc reached the maximum of $89.2 \%$. Although the conversion rate was not the highest, the reaction time was greatly reduced. In addition, we also reduced the production cost of enzyme by improving the expression level of secretion, which laid a foundation for the preparation of GlcNAc by enzymatic hydrolysis of colloidal chitin in industry. 


\section{CONCLUSION}

This study is the first report to identify the BlNagZ gene from $B$. licheniformis WX-02 and expressed in E. coli. The secretory expression level of BlNagZ is the highest among all the reported cases. After optimization the expression conditions, the highest activity of BlNagZ in the fermentation supernatant reached $13.62 \mathrm{U} / \mathrm{mL}$. Using high concentration colloidal chitin as substrate, the combination of recombinant BlNagZ and chitinase ChiA could produce GlcNAc with a yield of $89.2 \%$. The whole hydrolysis process was controlled within $3.5 \mathrm{~h}$, which is the fastest hydrolysis of colloidal chitin into GlcNAc speed at present.

\section{DATA AVAILABILITY STATEMENT}

The datasets presented in this study can be found in online repositories. The names of the repository/repositories and accession number(s) can be found below: https://www.ncbi.nlm. nih.gov/genbank/, CP012110.1.

\section{AUTHOR CONTRIBUTIONS}

SiJ and GZ designed the study. ShJ and CL completed the experiment and drafted the manuscript. CD and ZL provided

\section{REFERENCES}

Bruce, A. F., and Gounaris, K. (2006). Characterisation of a secreted N-acetyl-betahexosaminidase from Trichinella spiralis. Mol. Biochem. Parasitol. 145, 84-93. doi: 10.1016/j.molbiopara.2005.09.010

Chang, S., Guo, Y., Wu, B., and He, B. (2017). Extracellular expression of alkali tolerant xylanase from Bacillus subtilis lucky9 in E. coli and application for xylooligosaccharides production from agro-industrial waste. Int. J. Biol. Macromol. 96, 249-256. doi: 10.1016/j.ijbiomac.2016.11.032

Chen, J. K., Shen, C. R., and Liu, C. L. (2010). N-acetylglucosamine: production and applications. Mar. Drugs 8, 2493-2516. doi: 10.3390/md8092493

Chen, S., Liu, Z., Chen, J., and Wu, J. (2011). Study on improvement of extracellular production of recombinant Thermobifida fusca Cutinase by Escherichia coli. Appl. Biochem. Biotechnol. 165, 666-675. doi: 10.1007/s12010-011-9286-Z

Chen, S., Tong, X., Woodard, R. W., Du, G., Wu, J., and Chen, J. (2008). Identification and characterization of bacterial cutinase. J. Biol. Chem. 283, 25854-25862. doi: 10.1074/jbc.M800848200

Du, C., Jiang, S., Jiang, S., Zhou, Y., and Zhang, G. (2019). A Bacillus pumilus originated $\beta-\mathrm{N}$-acetylglucosaminidase for chitin combinatory hydrolysis and exploration of its thermostable mechanism. Int. J. Biol. Macromol. 132, 12821289. doi: 10.1016/j.ijbiomac.2019.04.054

Fang, S., Li, J., Long, L., Du, G., and Jian, C. (2011). Overproduction of alkaline polygalacturonate lyase in recombinant Escherichia coli by a two-stage glycerol feeding approach. Bioresour. Technol. 102, 10671-10678. doi: 10.1016/j. biortech.2011.09.020

Fu, X., Yan, Q., Yang, S., Yang, X., Guo, Y., and Jiang, Z. (2014). An acidic, thermostable exochitinase with $\beta$-N-acetylglucosaminidase activity fromPaenibacillus barengoltziiconverting chitin toN-acetyl glucosamine. Biotechnol. Biofuels 7:174. doi: 10.1186/s13068-014-0174-y

García-Fraga, B., Silva, A. F. D., López-Seijas, J., and Sieiro, C. (2015). A novel family 19 chitinase from the marine-derived Pseudoalteromonas tunicata CCUG 44952T: Heterologous expression, characterization and antifungal activity. Biochem. Eng. J. 93, 84-93. doi: 10.1016/j.bej.2014.09.014 technical support on experiment. YZ and $\mathrm{NH}$ helped to analyze the data. SiJ supervised the experiment process and revised the manuscript. GZ substantially revised the manuscript. All authors read and approved the final version of the manuscript.

\section{FUNDING}

This study was supported by the National Key R\&D Program of China (No. 2018YFA0901100), Natural Science Foundation of Hubei Province (2018CFA042), and 2016 Wuhan Yellow Crane Talents (Science) Program.

\section{SUPPLEMENTARY MATERIAL}

The Supplementary Material for this article can be found online at: https://www.frontiersin.org/articles/10.3389/fmicb. 2021.648373/full\#supplementary-material

Supplementary Figure 1 | Construction flow chart of recombinant expression vector pET26B-BINagZ.

Supplementary Figure 2 | Dynamics analysis of recombinant enzyme BINagZ.

Supplementary Figure $\mathbf{3}$ | The analysis of BINagZ secretion. Lane M, prestained protein ladder; Lane 1, fermentation supernatant without optimization; Lane 2, fermentation supernatant after optimization.

Supplementary Table 1 | Results of orthogonal experiment.

Huang, L., Wang, Q., Jiang, S., Zhou, Y., Zhang, G., and Ma, Y. (2016) Improved extracellular expression and high-cell-density fed-batch fermentation of chitosanase from Aspergillus fumigatus in Escherichia coli. Bioprocess Biosyst. Eng. 39, 1679-1687. doi: 10.1007/s00449-016-1643-4

Inokuma, K., Hasunuma, T., and Kondo, A. (2016). Ethanol production from $\mathrm{N}$-acetyl-d-glucosamine by Scheffersomyces stipitis strains. AMB Express 6:83. doi: 10.1186/s13568-016-0267-z

Jiang, S., Jiang, H., Zhou, Y., Jiang, S., and Zhang, G. (2019). High-level expression of $\beta$-N-acetylglucosaminidase BsNagZ in Pichia pastoris to obtain GlcNAc. Bioprocess Biosyst. Eng. 42, 611-619. doi: 10.1007/s00449-018-02067-5

Jorobekova, S., Kydralieva, K., and Hudaybergenova, E. (2005). "Influence of metal ions on the activity of soil humic-enzyme complexes," in Use of Humic Substances to Remediate Polluted Environments: From Theory to Practice, eds I. V. Perminova, K. Hatfield, and N. Hertkorn (Berlin: Springer-Verlag), 329341. doi: 10.1007/1-4020-3252-8_16

Kamps, J. J. A. G., Hopkinson, R. J., Schofield, C. J., and Claridge, T. D. W. (2019). How formaldehyde reacts with amino acids. Commun. Chem. 2:126. doi: 10.1038/s42004-019-0224-2

Kim, J.-S., Yoon, B.-Y., Ahn, J., Cha, J., and Ha, N.-C. (2015). Crystal structure of $\beta$-N-acetylglucosaminidase CbsA from Thermotoga neapolitana. Biochem. Biophys. Res. Commun. 464, 869-874. doi: 10.1016/j.bbrc.2015.07.053

Litzinger, S., Fischer, S., Polzer, P., Diederichs, K., Welte, W., and Mayer, C. (2010). Structural and kinetic analysis of Bacillus subtilis $\mathrm{N}$-acetylglucosaminidase reveals a unique Asp-His Dyad Mechanism*. J. Biol. Chem. 285, 35675-35684. doi: $10.1074 /$ jbc.M110.131037

Nguyen-Thi, N., and Doucet, N. (2016). Combining chitinase C and $\mathrm{N}$-acetylhexosaminidase from Streptomyces coelicolor A3(2) provides an efficient way to synthesize $\mathrm{N}$-acetylglucosamine from crystalline chitin. J. Biotechnol. 220, 25-32. doi: 10.1016/j.jbiotec.2015.12.038

Ogawa, M., Kitagawa, M., Tanaka, H., Ueda, K., Watsuji, T-o., Beppu, T., et al. (2006). A $\beta$-N-acetylhexosaminidase from Symbiobacterium thermophilum; gene cloning, overexpression, purification and characterization. Enzyme Microb. Technol. 38, 457-464. doi: 10.1016/j.enzmictec.2005.07.009 
Oliveira, E. S. D., Junges, Â, Sbaraini, N., Andreis, F. C., and Schrank, A. (2018). Molecular evolution and transcriptional profile of GH3 and GH20 $\beta-\mathrm{N}$-acetylglucosaminidases in the entomopathogenic fungus Metarhizium anisopliae. Genet. Mol. Biol. 41, 843-857. doi: 10.1590/1678-4685-gmb-20170363

Park, J. K., Kim, W. J., and Park, Y. I. (2015). Purification and characterization of an exo-type $\beta-\mathrm{N}$-acetylglucosaminidase from Pseudomonas fluorescens JK-0412. J. Appl. Microbiol. 110, 277-286. doi: 10.1111/j.1365-2672.2010. 04879.x

Patil, N. S., Waghmare, S. R., and Jadhav, J. P. (2013). Purification and characterization of an extracellular antifungal chitinase from Penicillium ochrochloron MTCC 517 and its application in protoplast formation. Process Biochem. 48, 176-183. doi: 10.1016/j.procbio.2012.11.017

Rosano, G. N. L., and Ceccarelli, E. A. (2014). Recombinant protein expression in Escherichia coli: advances and challenges. Front. Microbiol. 5:172. doi: 10.3389/ fmicb.2014.00172

Samant, S., Gunja, G., Karthikeyan, S., Haq, S. F., Nair, A., Sambasivam, G., et al. (2014). Effect of codon-optimized E. coli signal peptides on recombinant Bacillus stearothermophilus maltogenic amylase periplasmic localization, yield and activity. J. Ind. Microbiol. Biotechnol. 41, 1435-1442. doi: 10.1007/s10295014-1482-8

Shaoqing, Y., Shuang, S., Qiaojuan, Y., Xing, F., Zhengqiang, J., and Xinbin, Y. (2014). Biochemical characterization of the first fungal glycoside hydrolyase family $3 \beta-\mathrm{N}$-acetylglucosaminidase from Rhizomucor miehei. J. Agric. Food Chem. 62, 5181-5190. doi: 10.1021/jf500912b

Song, W., Zhang, N., Yang, M., Zhou, Y., He, N., and Zhang, G. (2020). Multiple strategies to improve the yield of chitinase a from Bacillus licheniformis in Pichia pastoris to obtain plant growth enhancer and GlcNA. Microbial Cell Factories 19:181. doi: 10.1186/s12934-020-01440-y

Talent, J. M., and Gracy, R. W. (1996). Pilot study of oral polymeric N-acetyl-dglucosamine as a potential treatment for patients with osteoarthritis. Clin. Ther. 18, 1184-1190. doi: 10.1016/S0149-2918(96)80073-7

Tsujibo, H., Hatano, N., Mikami, T., Hirasawa, A., and Miyamoto, K. (1998). A Novel $\beta-\mathrm{N}$-acetylglucosaminidase from Streptomyces thermoviolaceus OPC520: gene cloning, expression, and assignment to family 3 of the glycosyl hydrolases. Appl. Environ. Microbiol. 64, 2920-2924. doi: 10.1128/AEM.64.8. 2920-2924.1998

Ujiie, A., Nakano, H., and Iwasaki, Y. (2016). Extracellular production of Pseudozyma (Candida) antarctica lipase B with genuine primary sequence in recombinant Escherichia coli. J. Biosci. Bioeng. 121, 303-309. doi: 10.1016/j. jbiosc.2015.07.001

Wang, D., Li, A., Han, H., Liu, T., and Yang, Q. (2018). A potent chitinase from Bacillus subtilis for the efficient bioconversion of chitin-containing wastes. Int. J. Biol. Macromol. 116, 863-868. doi: 10.1016/j.ijbiomac.2018.05.122

Wei, X., Ji, Z., and Chen, S. (2010). Isolation of halotolerant Bacillus licheniformisWX-02 and regulatory effects of sodium chloride on yield and molecular sizes of poly- $\gamma$-glutamic acid. Appl. Biochem. Biotechnol. 160, 13321340. doi: 10.1007/s12010-009-8681-1

Yem, D. W., and Wu, H. C. (1976). Purification and properties of beta-Nacetylglucosaminidase from Escherichia coli. J. Bacteriol. 125, 324-331. doi: 10.1128/JB.125.1.324-331.1976

Zhou, J., Song, Z., Zhang, R., Ding, L., Wu, Q., Li, J., et al. (2016). Characterization of a NaCl-tolerant $\beta$-N-acetylglucosaminidase from Sphingobacteriumsp. HWLB1. Extremophiles 20, 547-557. doi: 10.1007/s00792-016-0848-4

Zhou, J., Song, Z., Zhang, R., Liu, R., Wu, Q., Li, J., et al. (2017). Distinctive molecular and biochemical characteristics of a glycoside hydrolase family 20 $\beta-\mathrm{N}$-acetylglucosaminidase and salt tolerance. BMC Biotechnology 17:37. doi: 10.1186/s12896-017-0358-1

Conflict of Interest: The authors declare that the research was conducted in the absence of any commercial or financial relationships that could be construed as a potential conflict of interest.

Copyright (C) $2021 \mathrm{Li}$, Jiang, Du, Lu, He, Zhou, Jiang and Zhang. This is an openaccess article distributed under the terms of the Creative Commons Attribution License (CC BY). The use, distribution or reproduction in other forums is permitted, provided the original author(s) and the copyright owner(s) are credited and that the original publication in this journal is cited, in accordance with accepted academic practice. No use, distribution or reproduction is permitted which does not comply with these terms. 